\title{
Reusable Elastomeric Respirators as An Alternative During the Covid-19 Pandemic: Hospital-Based Health Technology Assessment
}

\author{
Claudia Guevara Pérez ${ }^{1 *}$, Adriana Ávila Reina², Bibiana García Guarín ${ }^{3}$, Francy Cantor Cruz ${ }^{4}$, \\ Fredy Mendivelso Duarte ${ }^{5}$, Eduardo Low Padilla ${ }^{6}$, Nancy Yomayusa Gonzalez ${ }^{7,8}$, Emilio Herrera \\ Molina ${ }^{9}$, Edisson Romero Acosta ${ }^{7}$ and Carolina Coronado Benavides ${ }^{10}$ \\ ${ }^{1}$ Instituto Global de Excelencia Clínica Keralty, Centro de Evaluación de Tecnologías en salud, Departamento de gestión de dispositivos \\ médicos, Bogotá-Colombia. \\ ${ }^{2}$ Instituto Global de Excelencia Clínica Keralty, Centro de Evaluación de Tecnologías en salud, Departamento de estudios económicos, \\ Bogotá-Colombia.
}

${ }^{3}$ Agencia de Calidad y seguridad Keralty, Bogotá- Colombia.

${ }^{4}$ Instituto Global de Excelencia Clínica Keralty, Centro Iberoamericano de investigación en Salutogénesis y bienestar, Bogotá-Colombia.

${ }^{5}$ Instituto Global de Excelencia Clínica Keralty, Centro de Medicina basada e informada en la evidencia, Bogotá-Colombia.

${ }^{6}$ Instituto Global de Excelencia Clínica Keralty, Centro de Evaluación de Tecnologías en salud, Bogotá-Colombia.

${ }^{7}$ Instituto Global de Excelencia Clínica Keralty, Bogotá-Colombia.

${ }^{8}$ Grupo de Investigación Translacional-Fundación Universitaria Sanitas

${ }^{9}$ Keralty

${ }^{10}$ Purchasing department Keralty, Bogotá-Colombia

*Corresponding author: Claudia Guevara Pérez, Instituto Global de Excelencia Clínica Keralty, Centro de Evaluación de Tecnologías en salud, Departamento de gestión de dispositivos médicos, Av. Calle 127 \# 20 - 56 6th floor, Colombia

\section{ARTICLE INFO \\ Received: 㗀 August 19, 2020 \\ Published: 幽 September 09, 2020}

Citation: Claudia Guevara Pérez, Adriana Ávila Reina, Bibiana García Guarín, Francy Cantor Cruz, Fredy Mendivelso Duarte, et al. Reusable Elastomeric Respirators as An Alternative During the Covid-19 Pandemic: Hospital-Based Health Technology Assessment. Biomed J Sci \& Tech Res 30(2)2020. BJSTR. MS.ID.004910.

Keywords: Technology Assessment; Hospital-Based; Pandemics; Personal Protective Equipment; Respiratory Protective Devices; Mask

Abbreviations: PPE:Personal Protection Equipment; CDC: Centers for Disease Control; WHO: World Health Organization; RERs: Reusable Elastomeric Respirators; HTA: Health Technology Assessment; ICU: Intensive Care Unit; OSHA: Occupational Safety and Health Administration; NIOSH: National Institute for Occupational Safety and Health

\section{ABSTRACT}

Introduction: The pandemic caused by the novel coronavirus SARS-CoV-2 has increased the demand for health services and thereby created a shortage of personal protective equipment for health workers. In a real scenario of a shortage of N95 filtering facepiece respirators, organizations such as the Centers for Disease Control and Prevention recommend the alternative use of reusable elastomeric respirators.

objective: To perform a hospital-based health technology assessment of reusable half-face and full-face elastomeric respirators for use in intensive care units focused on patients with a confirmed or suspected diagnosis of COVID-19.

Method: The process was carried out with a multicriterion decision approach to assess six domains according to the recommendations of the European Network for Health Technology Assessment.

Results: The available evidence on reusable elastomeric respirators in healthcare settings is scarce. The studies found allow us to recommend the use of elastomeric respirators in a conditional context of a shortage of N95 respirators in specific areas, and our analysis shows that they are less expensive. These respirators are the first-line alternative to scarce $\mathrm{N} 95$ respirators and have cost advantages in the simulation scenario. A training, monitoring, and assessment strategy must be in place for the implementation of reusable elastomeric respirators. At the organizational level, the use of these respirators is perceived as safe and comfortable.

Conclusions: This hospital-based health technology assessment shows that reusable elastomeric respirators can be used in intensive care units as a rational alternative to disposable N95 respirators whose shortage is caused by a pandemic. 


\section{Introduction}

The severe acute respiratory syndrome coronavirus (SARSCoV-2)/coronavirus disease 2019 (COVID-19) pandemic has currently affected approximately $21,927,114$ people worldwide [1], of whom 775,000 have died and 13,917,917 have recovered, generating high demand for health services and personal protection equipment (PPE). Considering the mode of transmission of the virus, the PPEs recommended by the American Centers for Disease Control and Prevention (CDC) and the World Health Organization (WHO) are face shields or protective goggles, N95 filtering facepiece respirators commonly called "N95 respirators" (N95R) or higher-filter respirators, nonsterile gloves, and isolation gowns, which should be worn before entering the patient area and kept on at all times while working in potentially contaminated areas [2] This PPE is mostly single use. Given the uncertainty regarding the duration of the pandemic and the global increase in the demand for this and other PPE, a scenario of scarcity and potential shortage in the supply chain is a real and immediate concern. Specifically regarding N95R, the CDC has recommended alternatives for their optimization, such as prolonged use, reuse, and decontamination between uses. It has also recommended the alternative use of halffacepiece or full-facepiece reusable elastomeric respirators (RERs) [3-5] since they can protect against pathogens and other airborne hazards in healthcare settings.

RERs are frequently used at the industrial level as masks made of synthetic or natural rubber, which can be used, cleaned, disinfected, stored, and reused repeatedly. They are equipped with replaceable filter cartridges or flexible, disc-shaped, or pancakestyle filters or adequate air purification canisters, but this PPE requires maintenance and a supply of replaceable components that include straps, inhalation and exhalation valves, valve covers and filters, cartridges, and canisters [3]. Despite providing full protection of the eyes, nose, and mouth, RERs are not frequently used in the health field, which could hinder their acceptance and handling by health workers and require exhaustive training to achieve proper use at critical safety points such as donning, doffing, cleaning, and disinfecting these devices. However, the durability and reuse of these respirators make them desirable for health emergencies, in which the need for large quantities of respirators can be expected, as happened in previous health crises caused by Middle East respiratory syndrome, H1N1 influenza in 2009, and Ebola in 2014 [6,7]. The WHO defines health technology assessment (HTA) as the systematic evaluation of various aspects of "the application of organized knowledge and skills in the form of medicines, medical devices, vaccines, procedures and systems developed to solve a health problem and improve quality of life" in order to inform decision-making [8].

In the hospital context, studies of HTA are called hospital based HTAs, and they allow a local analysis of the implementation or not of a technology [9]. As in a regular HTA, aspects of clinical effectiveness and safety are evaluated. In some cases, the assessment is accompanied by an economic evaluation and other evaluations of interest to the hospital. On this background, the objective of this study was to evaluate the hospital-based health technology of half-facepiece and full-facepiece RERs as an alternative to the undersupplied N95R for use in the intensive care unit (ICU) for the care of patients with confirmed or suspected COVID-19.

\section{Methods}

The assessment was performed by the Technology Evaluation Center of an institution providing health services in Colombia, using a process with multicriterion decision approach to evaluate the domains suggested by the European Network for Health Technology Assessment and two additional domains suggested by the national HTA model [10]. In total, six domains were evaluated: health problem and current use of technology, description and technical characteristics of the technology, clinical effectiveness, safety, economic evaluation, and organizational aspects.

The methods used in the different domains are described below:

\section{Clinical Effectiveness and Safety}

Two reviewers systematically searched the literature independently, using as sources the Medline database; the regulatory agencies CDC, the National Institute for Occupational Safety and Health (NIOSH), the Occupational Safety and Health Administration (OSHA), and the ECRI Institute; and the gray literature on Google Scholar. The search terms used were elastomeric masks in health, elastomeric respirators, N95, pandemic, CoVID-19, health workers, and respiratory protection. The searches were limited to the English and Spanish languages and the period between May and July 2020.

\section{Economic Evaluation}

A three-stage health costing method was used: identification, measurement, and valuation of resources, with a time horizon of six months. In the identification stage, the human resources were estimated based on the workers required to care for 15 beds in an ICU. In the measurement stage, the RERs to be evaluated were full-facepiece and half-facepiece RERs. The gold standard for comparison was the combination of N95R + goggles + face shield. The filter types evaluated were the P100 series. In the valuation stage, the costs associated with the PPE were provided by local providers and were taken from the cost references of the Colombian health system.

\section{Organizational Aspects}

A perspective analysis and focus group were performed to evaluate the perception of health workers and decision-makers, respectively, regarding the use and inclusion of the RER technology. An implementation analysis was also performed based on the findings of the former. The perspective analysis was performed 
through an electronic survey with the objective of determining the perception of health workers about the safety, comfort, benefits, and limitations related to the use of RERs. A descriptive statistical analysis and narrative synthesis of the qualitative data were performed. Data collection was stopped when saturation in the qualitative responses was achieved. The focus group was conducted with hospital decision-makers, including managers, purchasing agents, and clinical experts in infectious diseases, intensive care, and dentistry. These positions were chosen for their potential role

Table 1: Technical description of the evaluated technologies.

\begin{tabular}{|c|c|}
\hline Technology & Technical characteristics \\
\hline N95R + goggles + face shield & $\begin{array}{c}\text { N95 respirators are disposable medical devices with or without a valve that filter } 95 \% \text { of particles smaller } \\
\text { than } 0.3 \mu \mathrm{m} \text {. Eye protection components such as goggles and a face shield are usually made of polymeric } \\
\text { materials }(11) .\end{array}$ \\
\hline Full-facepiece RER & $\begin{array}{l}\text { Provides total protection of eyes, nose, and mouth. The large lens provides a wide field of view for excellent } \\
\text { visibility. The lightweight, well-balanced design and silicone face seal provide good comfort, durability, and } \\
\text { ease of cleaning (12). Have a higher assigned protection factor (APF) (APF: 50) than N95 (APF: 10) (3). }\end{array}$ \\
\hline Half-facepiece RER & $\begin{array}{l}\text { Made of a softer silicone material, which provides greater comfort when used, especially over long periods. } \\
\text { Have the same filtration capacity as the full-facepiece RERs. Also require the use of goggles and face masks } \\
\text { (13). Have the same APF as N95R (APF: 10) (3). }\end{array}$ \\
\hline
\end{tabular}

\section{Effectiveness and Safety of RERs}

Experimental and simulation studies provide the main evidence on the use of RERs in the health context. A clinical, randomized, pragmatic, and multicenter study funded by the CDC evaluated the acceptance of and feasibility of rapidly training and fit testing RERs and 26 routine performance indicators in 153 participants. The RERs showed an advantage over N95R [12]. An observational study with eight subjects that evaluated the fit and tolerance of an RER with modifications to the original design approved by NIOSH found good tolerance to this device for 1 hour, which was the estimated time to perform an aerosol-generating procedure in the ICU [13]. Lastly, a descriptive study compared the technical characteristics of different respirators and concluded that RERs should be accompanied by 99- to 100-level filters in aerosol-generating procedures [14]. The studies agree on the recommendation that RERs are the first-line alternative to N95R in shortage scenarios given that they have the same safety and effectiveness advantages, conferred by the filtration, sealing, and comfort properties of the equipment when donned and doffed correctly and when they are properly reprocessed according to the recommendations of the CDC and NIOSH [6,15-17].

These respirators may also be a viable option for respiratory protection programs and for routine use in health care when logistic and implementation challenges are addressed, including personnel education and training and respirator cleaning, disinfection, and storage [7, 14], as well as adherence to institutional protocols. The review by Nardell et al. indicates that given the possibility of airborne contagion by contact with the respiratory droplets of asymptomatic individuals [17], a good clinical practice recommendation is to use a in incorporating the new technology in their work. The participants were informed in advance the results of the assessments of effectiveness and safety and the economic and regulatory aspects in the local context.

\section{Results}

\section{Technical Characteristics of the Technology}

The high-efficiency respirator types evaluated, and their technical characteristics are described in (Table 1).

surgical mask in addition to the RER in sterile and nonsterile areas [18]. However, the recommendations of the American College of Surgeons indicate that there is no quality evidence on the degree of protection and no contamination of sterile surgical fields from the use of RERs, even with the simultaneous use of surgical masks, emphasizing that the effectiveness of RERs depends mainly on three aspects: proper use, the fit and seal, and the filter performance [7]. At the local level, this technology is recommended as an alternative in a scenario of N95R shortage by the current Colombian consensus recommendations for the diagnosis, management, and treatment of infection by SARS-CoV-2/COVID-19 [18].

Regarding filtration capacity, the CDC recommends the use of NIOSH-certified RERs with a capacity equal to or greater than that of N95R for protection against inhalation of aerosolized contaminants in the hospital environment [4]. There are several filter types or series available for these respirators, classified according to their resistance to degradation by oil-based aerosols into the $\mathrm{N}, \mathrm{R}$, and $P$ series, and according to their ability to filter particles into the 95, 99, and 100 levels [4]. Depending on the conditions of use, the manufacturers propose filter replacement 40 hours to 30 days after the first use if there are no oil mists. However, in crisis situations, OSHA indicates that filters may be replaced "when necessary," for example, when they are damaged, dirty, contaminated, or obstructed $[3,19]$, given that in a hospital environment, the concentration of particles can be lower than in industrial environments. If the filter housing in different cartridge types is disinfected after each patient interaction, this could, according to the CDC recommendations in times of shortage and following OSHA protocols, allow use for up to one year [3]. 


\section{Economic Evaluation}

Based on the projection of the minimum number of health workers and conditions of use of the PPE, it was established that for an ICU with 15 beds at full capacity, a care team consisting of 78 people per 24 hours will be required (Annex 1). For these workers, the provision of reusable PPE, such as goggles, face shields, and respirators, and disposable PPE, including N95R and RER filters, was considered, the latter according to three replacement scenarios: at one, three, and six months. The N95R were assumed to be discarded after each shift.

Annex Table 1: Number of workers required in an intensive care unit per day.

\begin{tabular}{|c|c|}
\hline Worker & Number \\
\hline Head of nursing & 1 for every 3 beds \\
\hline Nursing assistant & 1 for every 2 beds \\
\hline Intensivists & 1 for every 7 beds \\
\hline Stretcher bearer & 1 stretcher bearer per shift \\
\hline Respiratory therapists & 1 for every 5 beds \\
\hline Specialist physician (per shift) & 3 per shift \\
\hline Nutritionist & 1 for every 15 beds \\
\hline Administrative and cleaning staff & 3 per shift \\
\hline Hours per shift & 8 hours \\
\hline Workers per shift & 26 \\
\hline Workers per day (24 hours) & 78 \\
\hline
\end{tabular}

\section{Comparative Cost Per Month Between the Alternatives} with Sensitivity Analysis

The estimated costs per month for the N95R (gold standard) and the RER are described in (Table 2). The unit value of each equipment is found in (Annex 1) [20]. The estimation of the base case was performed with the average costs (Annex 1). The highestcost strategy at six estimated months was the strategy with N95R, goggles, and face shield because it was the strategy in which the PPE were discarded after each use. Additionally, due to scarcity and high demand, the market price of N95R has fluctuated and at times even tripled. If the filter is changed every month, the full-facepiece RER strategy would cost \$USD 57,800. The lowest-cost strategy was the half-facepiece RER. According to the proposed scenarios, if filters are replaced every three or six months, the costs of the RER strategy in the ICU would be approximately $30 \%$ lower than those given in (Table 3). Given the fluctuation of local prices observed during the pandemic, a sensitivity analysis was performed on the prices of the alternatives.

Annex Table 2: Unit cost of the evaluated personal protective equipment (PPE).

\begin{tabular}{|c|c|c|}
\hline PPE & Price (average) & Price range: min - max \\
\hline N95 respirator & \$USD 4.08 & \$USD 2.76 - \$USD 8.27 \\
\hline Goggles & \$USD 2.66 & \$USD 1.38 - \$USD 4.14 \\
\hline Face shield & \$USD 5.05 & \$USD 2.10 - \$USD 9.51 \\
\hline $\begin{array}{c}\text { Full-facepiece } \\
\text { respirator }\end{array}$ & \$USD 99.8 & \$USD 90.51 - \$USD \\
173.68
\end{tabular}

Table 2: Estimated resources for six months of care.

\begin{tabular}{|c|c|c|c|c|c|c|c|c|c|}
\hline \multicolumn{10}{|c|}{ Replacement scenario } \\
\hline & \# workers & N95 & Goggles & Face shield & $\begin{array}{c}\text { Full-facepiece } \\
\text { RER* }\end{array}$ & $\begin{array}{c}\text { Half-facepiece } \\
\text { RER* }\end{array}$ & $\begin{array}{c}\text { Filters 1 } \\
\text { month }\end{array}$ & $\begin{array}{c}\text { Filters 3 } \\
\text { months }\end{array}$ & $\begin{array}{c}\text { Filters 6 } \\
\text { months }\end{array}$ \\
\hline Day & 78 & 78 & 78 & 78 & 78 & 78 & 0 & 0 & 0 \\
\hline Month & 78 & 2.340 & 78 & 78 & 78 & 78 & 78 & 78 & 78 \\
\hline 6 months & 78 & 14.040 & 78 & 78 & 78 & 78 & 468 & 156 & 78 \\
\hline \multicolumn{7}{|c|}{} \\
\hline
\end{tabular}

Table 3: Estimated cost for six months with monthly filter replacement (base case) (\$USD).

\begin{tabular}{|c|c|c|c|c|c|c|c|}
\hline & $\mathbf{1}$ month & $\mathbf{2}$ months & $\mathbf{3}$ months & $\mathbf{4}$ month & $\mathbf{5}$ months & $\mathbf{6}$ months & $\begin{array}{c}\text { Total expenditure in } \mathbf{6} \\
\text { months }\end{array}$ \\
\hline $\begin{array}{c}\text { N95 + goggles + face } \\
\text { shield }\end{array}$ & $\$ 10,149$ & $\$ 9,547$ & $\$ 9,547$ & $\$ 9,547$ & $\$ 9,547$ & $\$ 9,547$ & $\$ 57,885$ \\
\hline Full-facepiece RER & $\$ 9,333$ & $\$ 1,548$ & $\$ 1,548$ & $\$ 1,548$ & $\$ 1,548$ & $\$ 1,548$ & $\$ 17,075$ \\
\hline Half-facepiece RER & $\$ 3,357$ & $\$ 1,548$ & $\$ 1,548$ & $\$ 1,548$ & $\$ 1,548$ & $\$ 1,548$ & $\$ 11,100$ \\
\hline \multicolumn{7}{|c|}{ Exchange rate on July 21, 2020: \$COP 3,627.28 per \$USD 1.} \\
\hline
\end{tabular}


If the price of N95R returned to that before the pandemic (USD\$ 2.76) and the price of RERs doubled, the pattern of cost of the compared strategies would be maintained in the model: N95R were the highest-cost strategy, followed by full-facepiece and then halffacepiece RERs (Table 4). This analysis showed that the difference between the analyzed strategies was less than that estimated in the base case. Lastly, we estimated that for the N95R strategy to have the same cost as the full-facepiece RER alternative, the latter at the highest observed price, the price of N95R would have to be approximately USD 2.02 , close to $30 \%$ less than its cost before the pandemic.

Table 4: Sensitivity scenario: N95R at minimum price and respirators at maximum price (\$USD).

\begin{tabular}{|c|c|c|c|c|c|c|c|}
\hline & $\mathbf{1}$ month & $\mathbf{2}$ months & $\mathbf{3}$ months & $\mathbf{4}$ month & $\mathbf{5}$ months & $\mathbf{6}$ months & $\begin{array}{c}\text { Total expenditure } \\
\text { in } \mathbf{6} \text { months }\end{array}$ \\
\hline N95 + goggles + face shield & $\$ 7,060$ & $\$ 6,458$ & $\$ 6,458$ & $\$ 6,458$ & $\$ 6,458$ & $\$ 6,458$ & $\$ 39,352$ \\
\hline Full-facepiece RER & $\$ 16,106$ & $\$ 2,559$ & $\$ 2,559$ & $\$ 2,559$ & $\$ 2,559$ & $\$ 2,559$ & $\$ 28,903$ \\
\hline Half-facepiece RER & $\$ 5,749$ & $\$ 2,559$ & $\$ 2,559$ & $\$ 2,559$ & $\$ 2,559$ & $\$ 2,559$ & $\$ 18,546$ \\
\hline \multicolumn{7}{c}{ Exchange rate on July 21, 2020: \$COP 3,627.28 per \$USD 1. } \\
\hline
\end{tabular}

\section{Evaluation of Organizational Aspects}

A total of 14 health workers with experience in the use of RERs in the ICU and emergency departments during the COVID-19 pandemic were surveyed (Annex 2). Of these, 42.8\% (n=6) had used half-facepiece RERs, $50 \%(\mathrm{n}=7$ ) full-facepiece RERs, and $7.1 \%$ $(\mathrm{n}=1)$ both. A total of $92.8 \%(\mathrm{n}=13)$ reported having received training before use the RERs; 92.8\% ( $n=13$ ) considered RERs more comfortable than N95R and 7.1\% $(n=1)$ equally comfortable. Regarding safety, $64.2 \%(n=9)$ considered that RERs are safer than N95R, and the other $35.7 \%(n=5)$ considered them equally safe. All 14 of the respondents would recommend RERs as an alternative to N95R. The perception of comfort was related to the characteristics of the technology. These characteristics included the fact that RERs are easy to use and reusable, the seal is easily checked, they do not fog up during care activities, they allow the workers to breathe better, they are lightweight, they do not heat up, they do not squeeze, and they do not mark the skin when fitted right. RERs are also perceived as safer when performing orotracheal intubation in patients with COVID19, which increases comfort.

Annex 2: Survey of health worker perceptions.

1. What type(s) of elastomeric respirator(s) have you used? Half-facepiece elastomeric respirator/Full-facepiece elastomeric respirator/Both

2. Before using elastomeric respirators, were you trained in their use, maintenance, and cleaning? YES/NO

3. Regarding the comfort of elastomeric respirators, you consider them: More comfortable than N95 respirators/Less comfortable than N95 respirators/As comfortable as N95 respirators.

4. Regarding the perception of the safety of elastomeric respirators for health workers, you consider them: Safer than N95 respirators/Less safe than N95 respirators/As safe as N95 respirators.
5. What benefits have you found in the use of this type of respirator? (Open answer)

6. What difficulties or limitations have you encountered in the use of this type of respirator? (Open answer)

7. Do you recommend elastomeric respirators as an alternative to disposable N95 respirators? YES/NO

In regard to the perception of safety, although the respondents mentioned the lack of evidence on their superiority over N95R, they reported the same perceptions as for comfort, i.e., good sealing and precision, making them feel more safe. The respondents were also emphatic about the need to disinfect and clean the RERs before each use. Additional benefits reported beyond the perceived comfort and safety included a calm feeling during the performance of procedures; greater durability, which they considered costeffective; odor control provided by the filters and the fact that the same air is not reinhaled; and self-protection due to the barrier preventing the workers from touching their own mouth and eyes reflexively. The difficulties related to the use of RERs included challenges in logistical and implementation aspects, such as the shortage of replacement filters, ensuring adequate cleaning of the RER, and having a place to store them. Regarding use, only difficulty verbally communicating with other workers or patients was mentioned. The decision-makers in general considered the RERs an appropriate alternative, and they endorsed their use as an alternative during possible shortages.

They reported a desire to conduct a pilot study in some services within an institutional program that would include monitoring of the proper use, cleaning, disinfection, and maintenance of these reusable devices. Lastly, regarding the implementation of RER use, the correct use and performance of this technology must be ensured by:

1. Training: Focused on achieving a proper fit and sealing of the face. OSHA regulations require a written respiratory 
protection program that includes fit testing and seal checking by the user each time the RER is used $[3,4,21]$.

2. Standardized cleaning and disinfection protocols, validated and in compliance with the manufacturer's recommendations: The different elastomeric components of the respirator must be cleaned and disinfected using proper procedures and considering their potential impact on the respirator mask components, taking care not to damage the filter in this process. It is recommended that cleaning and disinfection be standardized and centralized and not performed by different workers after using the RERs $[3,4]$. Bessesen et al. showed that when cleaning and disinfection protocols were not standardized and were performed by personnel not trained for this activity, a significantly higher number of mistakes were made [22].

3. Adequate maintenance and replacement of components when necessary: This is particularly relevant for filters. In addition, personnel should be trained to perform the required maintenance, including storage, inspection, distribution, repair, or replacement of parts, as well as cleaning, disinfection, and disposal $[3,4]$.

\section{Discussion}

The COVID-19 pandemic declared in March 2020 by the WHO [25] has led to the design of various strategies for reducing its rate of spread, strengthening health systems, sensitizing the population, and carrying out prevention actions, among others. Together with these measures and the easy transmissibility of the SARS-CoV-2 virus, health workers are at high risk of contagion, which requires us to strengthen all biosecurity measures and has caused a high demand for PPE that has exceeded the worldwide supply of such equipment, in particular for respiratory protection such as N95R. Hospital-based HTAs are tools to inform decision-making at the local and specific level of health service providers and are a useful undertaking in the current global health crisis. The present HTA shows that despite the lack of evidence in hospital settings, RERs are seen as a viable first-line alternative to the shortage or limited availability of N95R $[3,6]$, especially in the ICU. The acceptance and use experience reported by different health workers during the pandemic and the technical attributes of their quality, safety, and efficacy (already validated by international agencies) make them the best alternative in a scenario of N95R scarcity.

The use of RERs both in the ICU and potentially in other hospital services conditions their use on the existence of a hospital implementation program that guarantees training and monitoring of the use of this PPE and involves a standardized and centralized process for RER cleaning, disinfection, storage, and maintenance by qualified personnel [7]. Additional aspects should be evaluated in other services, such as in operating rooms, for which the American College of Surgeons recommends the use of RERs only in repeated-exposure circumstances during an N95R shortage due to a pandemic, accompanied by a surgical mask, because there is concern that unfiltered air leaving the exhalation valve can potentially contaminate the sterile field [22]. Our cost analysis in the ICU showed that the difference between the half- or fullfacepiece RER option versus N95R is high, that is, RERs are a less expensive alternative even if the price of N95R is lowered to the pre-pandemic price. This conclusion is similar to that drawn by others [24]. Although the data are shown in US dollars, they may vary in different countries; thus, it is advisable to introduce RERs early in institutions by combining the use of the N95R and RER PPE.

\section{Conclusions}

This hospital-based HTA shows that despite the lack of evidence in hospital settings, RERs are a viable, lower-cost alternative and could be the first-line alternative in scenarios of N95R shortage or limited availability, especially in the ICU during the SARS-CoV-2/ COVID-19 pandemic. The clinical efficacy, safety, and effectiveness of the implementation of this technology in other services, as well as its economic implications, should be re-evaluated according to the dynamics of care, the nature of the services, and the required personnel. Further studies are necessary, and given the current pandemic scenario, they should be carried out to generate quality evidence on the safety, efficacy, and effectiveness of the RER technology.

\section{Acknowledgements}

None.

\section{Conflicts of Interests}

The authors declare no conflict of interest.

\section{Author's Contributions}

Claudia Guevara Pérez, Adriana Ávila Reina, Bibiana García Guarín, Francy Cantor Cruz: They contributed to the study design, the data collection and analysis, the writing of the first draft and subsequent drafts of the manuscript and approval of the final version of this document. Nancy Yomayusa Gonzalez, Eduardo Low Padilla, Fredy Mendivelso Duarte, Edisson Romero Acosta, Carolina Coronado Benavides: They contributed to the study design, the data collection and analysis, critical review of the first draft and subsequent drafts of the manuscript and approval of the final version of this document.

\section{Funding}

None.

\section{References}

1. (2020) Johns Hopkins University. COVID-19 Dashboard by the Center for Systems Science and Engineering (CSSE).

2. (2020) Centers for Disease Control and Prevention. Using Personal Protective Equipment (PPE). 
3. (2020) Centers for Disease Control and Prevention. Elastomeric Respirators: Strategies During Conventional and Surge Demand Situations.

4. (2020) Centers for Disease Control and Prevention. Respirator TrustedSource Information, Section 1: NIOSH-Approved Respirators.

5. (2020) ECRI Institute. Safety of Extended Use and Reuse of N95 Respirators, Clinical Evidence Assessment.

6. Duling MG, Lawrence RB, Slaven JE, Coffey CC (2007) Simulated workplace protection factors for half-facepiece respiratory protective devices. J Occup Environ Hyg (6): 420-431.

7. (2020) The National Academies of Science Engineering and Medicine. Reusable elastomeric respirators in health care: Considerations for routine and surge use. Washington: The National Academies Press.

8. (2020) World Health Organization. Health technology assessment.

9. Halmesmäki E, Pasternack I, Roine R (2016) Hospital-based health technology assessment (HTA) in Finland: a case study on collaboration between hospitals and the national HTA unit. Health Res Policy Syst 14: 25.

10. (2016) European network for Health Technology Assessment. HTA Core Model $\AA$.

11. (2020) Centers for Disease Control and Prevention. Strategies for Optimizing the Supply of Eye Protection.

12. Pompeii LA, Kraft CS, Brownsword EA, Lane MA, Benavides E, et al. (2020) Training and Fit Testing of Health Care Personnel for Reusable Elastomeric Half-Mask Respirators Compared with Disposable N95 Respirators. JAMA 323(18): 1849-1852.

13. Lawrence RB, Duling MG, Calvert CA, Coffey CC (2006) Comparison of Performance of Three Different Types of Respiratory Protection Devices. J Occup Environ Hyg 3(9): 465-474.

14. Mick P, Murphy R (2020) Aerosol-generating otolaryngology procedures and the need for enhanced PPE during the COVID-19 pandemic: A literature review. J Otolaryngol Head Neck Surg 49(1): 29.

\section{ISSN: 2574-1241}

DOI: $10.26717 /$ BJSTR.2020.30.004910

Claudia Guevara Pérez. Biomed J Sci \& Tech Res

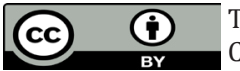

This work is licensed under Creative Commons Attribution 4.0 License

Submission Link: https://biomedres.us/submit-manuscript.php
15. Howard BE (2020) High-Risk Aerosol-Generating Procedures in COVID-19: Respiratory Protective Equipment Considerations. Otolaryngol Head Neck Surg 163(1): 98-103.

16. Liu DCY, Koo TH, Wong JKK, Wong YH, Fung KSC, et al. (2020) Adapting re-usable elastomeric respirators to utilise anaesthesia circuit filters using a 3D-printed adaptor - a potential alternative to address N95 shortages during the COVID-19 pandemic. Anaesthesia 75(8): 10221027.

17. Nardell EA, Nathavitharana RR (2020) Airborne Spread of SARS-CoV-2 and a Potential Role for Air Disinfection. JAMA 324(2): 141-142.

18. (2020) Grupo ACIN- IETS. Consenso Colombiano de atención, diagnóstico y manejo de la infección por SARS-CoV-2/Covid -19 en establecimientos de atención de la salud. Segunda Edición. Recomendaciones basadas en consenso de expertos e informadas en la evidencia. Infectio 24(3).

19. (2020) World Health Organization. Uso racional del equipo de protección personal frente a la COVID-19 y aspectos que considerar en situaciones de escasez graves: orientaciones provisionales, 6 de abril de 2020.

20. (2020) Colombia compra eficiente. Catálogo de Elementos de Protección Personal(EPP).

21. Vo E, Zhuang Z, Horvatin M, Liu Y, He X, et al. (2015) Respirator Performance against Nanoparticles under Simulated Workplace Activities. Ann Occup Hyg 59(8): 1012-1021.

22. (2020) Occupational Safety and Health Administration. Hospital Respiratory Protection Program Toolkit. Resources for Respirator Program Administrators.

23. (2020) World Health Organization. WHO Director-General's opening remarks at the media briefing on COVID-19 - 11 March 2020.

24. Baracco G, Eisert S, Eagan A, Radonovich L (2015) Comparative Cost of Stockpiling Various Types of Respiratory Protective Devices to Protect the Health Care Workforce During an Influenza Pandemic. Disaster Med Public Health Prep 9(3): 313-318.

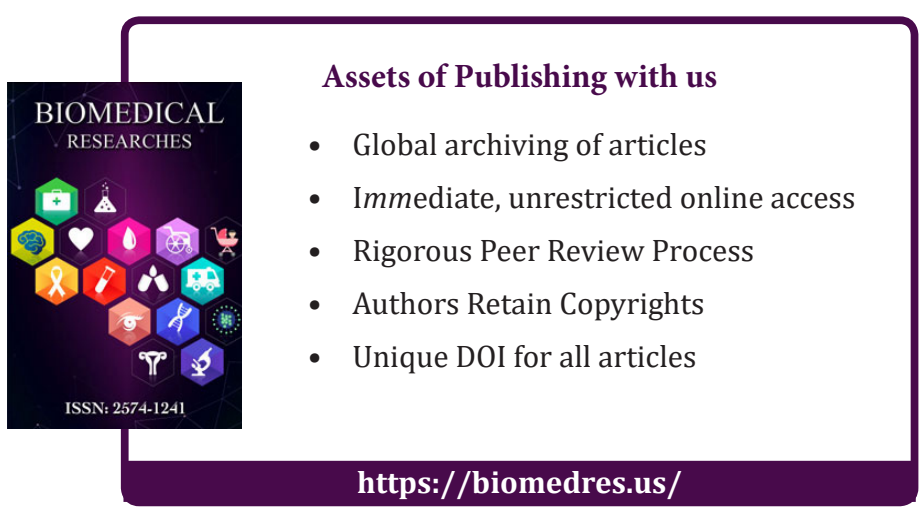

